\title{
Granzyme B releases vascular endothelial growth factor from extracellular matrix and induces vascular permeability
}

\author{
Alon Hendel ${ }^{1,2}$, Ivy Hsu ${ }^{1,2}$ and David J Granville $e^{1,2}$
}

The formation of unstable, leaky neovessels underlies the pathogenesis of many chronic inflammatory diseases. Granzyme B (GZMB) is an immune-derived serine protease that accumulates in the extracellular matrix (ECM) during chronic inflammation and is capable of cleaving fibronectin (FN). Vascular endothelial growth factor (VEGF) is a potent vascular permeabilizing agent that is sequestered in the ECM through its interaction with FN. As GZMB levels are elevated in chronic inflammatory diseases that are associated with increased vascular permeability, the role of GZMB in the regulation of VEGF bioavailability and vascular permeability were assessed. GZMB was added to either VEGF bound to FN or VEGF bound to endothelial cell (EC)-derived ECM. Supernatants containing released VEGF were assessed to determine VEGF activity by treating EC and evaluating VEGF receptor-2 (VEGFR2) phosphorylation. GZMB released VEGF from both FN and from EC-derived matrix, whereas GZMB inhibition prevented FN cleavage and VEGF release. GZMB-mediated VEGF release resulted in significant phosphorylation of VEGFR2. The role of GZMB-mediated VEGF release in altering vascular permeability was also assessed in vivo using Miles/Evans blue permeability assay. GZMB induced a significant VEGF-dependent increase in vascular permeability in vivo that was reduced in the presence of an anti-VEGF-neutralizing antibody. Inflammatory-mediated vascular leakage was also assessed in GZMB-KO mice using a delayed-type hypersensitivity model. GZMB-KO mice exhibited reduced microvascular leakage compared with $\mathrm{C} 57 \backslash \mathrm{B} 6$ controls. GZMB increases vascular permeability in part through the proteolytic release of ECM-sequestered VEGF, leading to VEGFR2 activation and increased vascular permeability in vivo. These findings present a novel role for GZMB as a modulator of vascular response during chronic inflammation.

Laboratory Investigation (2014) 94, 716-725; doi:10.1038/labinvest.2014.62; published online 5 May 2014

KEYWORDS: fibronectin; granzyme B; inflammation; vascular permeability; VEGF

Increased vascular permeability is one of the earliest manifestations of inflammation resulting in extravasation of protein-rich plasma into the affected tissue. Acute vascular permeability allows the deposition of circulating plasma matrix proteins including fibrin and fibronectin (FN) that facilitate cell migration into the inflamed area. ${ }^{1}$ This process also provides an access point for immune cells and immunoglobulins to enter the tissue and help fight foreign antigens. $^{2}$ In contrast, chronic vascular hyperpermeability is suggested to sustain the inflammatory response and delay resolution, further exacerbating chronic inflammation., ${ }^{2,3}$
This type of vascular hyperpermeability underlies the pathogenesis of a large number of chronic disorders including rheumatoid arthritis (RA), psoriasis, ocular disease, cancer, and chronic wounds. ${ }^{2,3}$

Vascular endothelial growth factor (VEGF) is a potent vascular permeabilizing agent that is highly expressed during chronic inflammation and contributes to pathological angiogenesis. ${ }^{4}$ VEGF induces vascular leakage by activating VEGF receptor 2 (VEGFR2)-mediated signaling events in endothelial cells (ECs), leading to plasma extravasation. ${ }^{5}$ Cellular sources for VEGF during inflammation may include

\footnotetext{
${ }^{1}$ Centre for Heart Lung Innovation, St Paul's Hospital, Vancouver, BC, Canada and ${ }^{2}$ Departments of Pathology and Laboratory Medicine, University of British Columbia, Vancouver, BC, Canada

Correspondence: Professor DJ Granville, PhD, Centre for Heart Lung Innovation, St Paul's Hospital, University of British Columbia, Room 166, Burrard Building, 1081 Burrard Street, Vancouver, BC V6Z 1Y6, Canada.

E-mail: David.Granville@hli.ubc.ca

Received 23 September 2013; revised 11 March 2014; accepted 20 March 2014
} 
macrophages and mast cells; however, it can also be expressed by ECs and acts in a paracrine and autocrine manner. ${ }^{6}$

The extracellular matrix (ECM) has a major role in regulating VEGF bioavailability. VEGF contains a cluster of basic residues that facilitate its interaction with anionic ECM proteins. ${ }^{7}$ VEGF interaction with the ECM greatly determines its bioavailability as the majority of VEGF is retained in the ECM after cell secretion. ${ }^{7}$ Other proteases may alter VEGF interaction with the ECM including plasmin ${ }^{7}$ and MMPs, ${ }^{8,9}$ giving rise to increased microvessel leakage and formation of aberrant neovasculature that is characteristic of pathological angiogenesis. ${ }^{8}$ Thus, ECMsequestered VEGF can be liberated by proteases and this process has significant implications on vascular integrity. Importantly, VEGF binds to the growth factor binding domain in FN. ${ }^{10-12}$ Binding of VEGF to FN enhances EC migration, proliferation, and promotes angiogenesis. ${ }^{10,11,13}$ However, the consequences of VEGF release from FN by a specific protease and its impact on vascular integrity has yet to be explored.

Granzyme B (GZMB) is a serine protease that is primarily expressed by immune cells including lymphocytes, macrophages, and mast cells. ${ }^{14}$ Although traditionally viewed as a proapoptotic intracellular protease used by cytotoxic lymphocytes to induce target cell apoptosis, we and others have suggested an important role of extracellular GZMB activity in a number of chronic inflammatory diseases (reviewed in Boivin et al. ${ }^{15}$ and Hiebert and Granville ${ }^{16}$ ). GZMB accumulates in the ECM of inflamed tissues and is present in several bodily fluids in chronic diseases such atherosclerosis, chronic obstructive pulmonary disease (COPD), and RA. ${ }^{17,18}$ ECM proteolysis by GZMB leads to impaired tissue integrity in diseases. In a mouse model of abdominal aortic aneurysm (AAA), GZMB cleavage of fibrillin-1 and decorin results in increased AAA rupture. ${ }^{19,20}$ In addition, degradation of decorin and FN by GZMB leads to increased skin thinning and delayed wound healing in apolipoprotein E-knockout (KO) mice. ${ }^{21,22}$ Moreover, extracellular GZMB is suggested to potentiate proinflammatory cytokine activities as it converts the pro form of IL-18 to its active form, leading to increased IFN- $\gamma$ release from human keratinocytes. ${ }^{23}$ GZMB cleavage of IL- $1 \alpha$ results in enhanced proinflammatory activity of this cytokine both in vitro and in vivo. ${ }^{24}$ Interestingly, GZMB cleavage of decorin, biglycan, and betaglycan leads to release of TGF- $\beta 1$ from the matrix, suggesting that GZMB may indirectly affect normal cell function by altering growth factor bioavailability. ${ }^{25}$

We have previously demonstrated that GZMB cleavage of FN dysregulates angiogenesis by impairing EC adhesion, migration, and capillary formation. ${ }^{26}$ The present study explores the effects of GZMB-mediated FN proteolysis on VEGF bioavailability and activity. We hypothesized that GZMB-mediated FN cleavage releases VEGF from the ECM and promotes vascular leakage.

\section{MATERIALS AND METHODS VEGF Release Assay}

The 48-well plates were coated with human purified plasma FN $(20 \mu \mathrm{g} / \mathrm{ml})$ (Sigma, St Louis, MO, USA) in DPBS for $1 \mathrm{~h}$ at $37^{\circ} \mathrm{C}$. Wells were then blocked with $1 \%$ BSA in DPBS for $30 \mathrm{~min}$ at $37^{\circ} \mathrm{C}$. VEGF $165(50 \mathrm{ng} / \mathrm{ml} ; \mathrm{R} \& \mathrm{D}$ Systems, Minneapolis, MN, USA) was added to the FN-coated wells and incubated for $2 \mathrm{~h}$ at $37^{\circ} \mathrm{C}$ followed by extensive washing to remove unbound VEGF. Human purified GZMB (50 nM; Axxora, San Diego, CA, USA) in Tris buffer (50 mM Tris, $\mathrm{pH}=7.4)$ with either vehicle control (1:100 DMSO) or GZMB inhibitor (Compound $20(50 \mu \mathrm{M})$, UBC Centre for Drug Research and Development, Vancouver, BC, Canada ${ }^{27}$ were added to the well for additional $2 \mathrm{~h}$ at $37^{\circ} \mathrm{C}$. Supernatants were analyzed by VEGF ELISA (R\&D Systems) according to the manufacturer's instructions. For VEGF release from human umbilical vein endothelial cell (HUVEC) matrix, HUVECs were cultured in a six-well plate and grown to confluence in complete growth media (EGM2 + 2\% FBS; Clonetics/Lonza, Walkersville, MD, USA). Cells were maintained in serum-reduced ( $0.2 \%$ FBS) media for 9 days and media were changed every 2 days. To remove the cells while leaving the ECM intact, cells were washed 3 times with DPBS and $200 \mu \mathrm{l} /$ well of $0.25 \mathrm{M}$ ammonium hydroxide was added and incubated for $20 \mathrm{~min}$ at RT. Wells were washed 3 times with $\mathrm{dH}_{2} \mathrm{O}$ and cell removal was confirmed by microscopical examination. Remaining ECM was then blocked with $1 \%$ BSA for $30 \mathrm{~min}$ at $37^{\circ} \mathrm{C}$, followed by addition of VEGF $(50 \mathrm{ng} / \mathrm{ml})$ in $1 \%$ BSA for $2 \mathrm{~h}$ at $37^{\circ} \mathrm{C}$. Unbound VEGF was removed by washing the wells with DPBS and GZMB ( $50 \mathrm{nM})$ in Tris buffer with either vehicle control or Compound 20 (UBC Centre or Drug Research and Development) were added to the well for additional $2 \mathrm{~h}$ at $37^{\circ} \mathrm{C}$. Supernatants were analyzed by VEGF ELISA.

\section{FN and VEGF Cleavage Assay}

The 48-well plates were coated with FN as described above. GZMB (50 nM), plasmin (50 nM), GZMB (50 nM) + Compound $20 \quad(50 \mu \mathrm{M})$, plasmin $(50 \mathrm{nM})+$ aprotinin $(125 \mathrm{nM}), \mathrm{GZMB}(50 \mathrm{nM})+$ aprotinin (Sigma) were added to the wells in Tris buffer for $2 \mathrm{~h}$ at $37^{\circ} \mathrm{C}$ (plasmin was kindly provided by Dr Ed Pryzdial, University of British Columbia). Enzyme preparations from the above experiment were used for VEGF cleavage assay. GZMB $(50 \mathrm{nM})$ or plasmin $(50 \mathrm{nM})$ were incubated with $100 \mathrm{ng}$ VEGF in Tris buffer for $2 \mathrm{~h}$ at $37^{\circ} \mathrm{C}$. Supernatants from the FN cleavage assay and the VEGF cleavage assay samples were analyzed by western blotting. In brief, samples were resolved by either 10\% (for FN supernatants) or $20 \%$ (for VEGF samples) SDS-PAGE and gels were transferred to PVDF membranes. Membranes were blocked with $2.5 \%$ milk and immunoblotted using antihuman FN Ab (R\&D) in a 1:1000 dilution in blocking solution or biotinylated anti-human VEGF (R\&D Systems) in a 1:100 dilution overnight at $4{ }^{\circ} \mathrm{C}$. FN signal was detected by 
incubating membrane with IRDye 800 anti-mouse Ab (LICOR Biosciences, Lincoln, NE, USA) for $1 \mathrm{~h}$ at RT and fluorescent signal was imaged using the Li-COR Odyssey Infrared Imaging System (Li-COR Biosciences). VEGF signal was detected by first incubating membrane with $\mathrm{ABC}$ reagent (Vector Laboratories, Burlingame, CA, USA) for $30 \mathrm{~min}$ at RT and adding ECL Plus C) substrate (GE Healthcare, Buckinghamshire UK). Chemiluminescence signal was captures using Chemigenius Bioimaging System (Syngene, Frederick, MD, USA). Mouse FN cleavage assay was performed in the same way as described for human FN, using 48 culture wells coated with mouse FN $(20 \mu \mathrm{g} / \mathrm{ml}$; Sigma) treated with either recombinant mouse granzyme B (mGZMB; Sigma) or human GZMB.

\section{VEGFR2 Phosphorylation by GZMB-Mediated VEGF Release from FN}

HUVECs were grown to confluence in six-well plate in complete growth media (EGM2 $+2 \%$ FBS). Media were changed to EGM2 no VEGF + 1\% FBS media for 2 days before treating cells with VEGF release supernatants. VEGF release from FN-coated wells was performed as described above, but this time treatments were prepared in M199 media (HyClone, Logan, UT, USA) to allow the use of the supernatants as a treatment media for HUVEC culture. VEGF release supernatants were added to HUVEC culture for $7 \mathrm{~min}$ followed by rapid cell lysis with lysis buffer (CellLytic M, Sigma) that included phosphatase inhibitor cocktail (1:100) and proteinase inhibitor cocktail (1:100; Sigma). Total protein concentration was quantified using Bio-Rad Bradford protein assay (Bio-Rad Laboratories), and $50 \mu \mathrm{g}$ of total protein cell lysates were resolved by 10\% SDS PAGE and immunoblotted using pVEGFR2y1214 Ab, pVEGFR2y1175 $\mathrm{Ab}$, and total VEGFR2 Ab (Cell Signaling Technology, Danvers, MA, USA; 1:700 dilution in $2.5 \%$ milk). $\beta$-Tubulin immunoblotting was used as a loading control. Densitometry was used to quantify pVEGFR2 and values were divided by the expression levels of total VEGFR2 and normalized to $\beta$-tubulin loading control $(n=3$ with three replicates per treatment).

\section{In Vivo Permeability Assay}

Animal procedures described were performed in accordance with the appropriate University of British Columbia (UBC) guidelines for animal experimentation and approved by the UBC Animal Care Committee. CD1 female mice, 8-10 weeks old, were injected with $200 \mu$ l Evans blue dye $(0.5 \%$ in injectable saline; Sigma) via the tail vein. The following treatments were prepared in injectable saline to a final volume of $10 \mu \mathrm{l}$ ( $n=5$ for each treatment group): mGZMB (100 ng), mGZMB (100 ng) + neutralizing anti-mouse VEGF antibody (1.5 $\mu \mathrm{g}$; R\&D Systems), mGZMB (100 ng) + goat IgG control ( $1.5 \mu \mathrm{g} ; \mathrm{R} \& \mathrm{D}$ Systems). Treatments were injected intradermally to the ear. After $30 \mathrm{~min}$, mice were killed and perfused with saline to clear the circulation from any remaining dye. Ear tissues were excised using a $7 \mathrm{~mm}$ punch biopsy and dried in $60^{\circ} \mathrm{C}$ incubator overnight. Tissues were weighed and dye was extracted by incubating tissues with $300 \mu$ l formamide (Sigma) in $60^{\circ} \mathrm{C}$ incubator for $24 \mathrm{~h}$. Absorbance of the extracted dye was measured at $610 \mathrm{~nm}$ and values were normalized to tissue weight.

\section{VEGF, FN Immunohistochemistry, and Immunofluorescence}

Formalin-fixed, paraffin-embedded sections of untreated mouse ear tissue were sectioned at $5 \mu \mathrm{m}$ thickness. Sections were deparaffinized and rehydrated in xylene and decreasing concentrations of ethanol. Antigen retrieval was performed by boiling slides in citrate buffer $(\mathrm{pH}=6)$ (Invitrogen, Carlsbad, CA, USA) for $15 \mathrm{~min}$ followed by 30 min cooling at room temperature. Endogenous peroxides were quenched by incubating sections in $3 \% \mathrm{H}_{2} \mathrm{O}_{2}$ for $15 \mathrm{~min}$. Sections were blocked with $10 \%$ rabbit serum for $30 \mathrm{~min}$ and incubated overnight at $4{ }^{\circ} \mathrm{C}$ with $10 \mu \mathrm{g} / \mathrm{ml}$ goat anti-mouse VEGF $\mathrm{Ab}$ (R\&D Systems). Sections were incubated with 1:350 dilution of biotinylated rabbit anti-goat $\mathrm{Ab}$ for 30 min followed by signal amplification using Vectastain $A B C$ system (Vector Laboratories) according to the manufacturer's instructions. Staining was visualized using DAB (Vector Laboratories). Images were captured using Nikon Eclipse E600 microscope (Nikon). Mouse kidney sections were used as positive control for VEGF staining. Negative control samples undergo the same treatment without primary Ab incubation. For double immunofluorescence staining for VEGF and FN, untreated mouse ear tissue sections were blocked with $10 \%$ donkey serum for $1 \mathrm{~h}$ and coincubated overnight at $4{ }^{\circ} \mathrm{C}$ with goat anti-mouse VEGF Ab (R\&D Systems) and rabbit anti-FN Ab (Abcam, Cambridge, MA, USA) diluted 1:20 and 1:500, respectively. Sections were then incubated for $1 \mathrm{~h}$ with donkey anti-goat Alexa $594 \mathrm{Ab}$ for VEGF detection, and with donkey anti-rabbit Alexa $488 \mathrm{Ab}$ for FN detection (1:350 dilution, Invitrogen). Fluorescent images were captured using Leica Upright Fluorescence Microscope with Fast Confocal Scanner and CCD camera (Leica Microsystems, Wetzlar, Germany).

\section{In Vivo Oxazolone-Induced Delayed-Type Hypersensitivity (DTH) Reaction Model}

Animal procedures described were performed in accordance with the appropriate UBC guidelines for animal experimentation and approved by the UBC Animal Care Committee. On day 1, 8-11-week-old C57/BL6 wild-type (WT) and GZMB-KO mice were sensitized by applying $2.5 \%$ oxazolone (Sigma-Aldrich, St Louis, MO, USA) dissolved in 4:1 acetone/olive oil topically on shaved abdomen $(50 \mu \mathrm{l})$ and each paw $(25 \mu \mathrm{l})$. On day 7 , mice were challenged by applying $1 \%$ oxazolone dissolved in $4: 1$ acetone/olive oil topically to each ear $(15 \mu \mathrm{L})$. Vascular permeability was examined $24 \mathrm{~h}$ (WT, $n=8$; GZMB-KO, $n=11$ ) and $72 \mathrm{~h}$ (WT, $n=11$; GZMB-KO, $n=7$ ) after challenging by injecting the mice 
with $400 \mu$ l Evans blue dye (1\% in injectable saline) via the inferior vena cava. After $15 \mathrm{~min}$, mice were killed and ear tissue collections and analysis were performed as described for the in vivo permeability assay.

\section{Statistics}

One-way analysis of variance (ANOVA) with Bonferroni's multiple comparison post test was performed to determine statistical differences between multiple groups. A paired $t$-test was performed to determine statistical difference between two matching groups. A value of $P<0.05$ was considered significant.

\section{RESULTS}

\section{GZMB Releases VEGF from Human Plasma FN}

To examine the ability of GZMB to release VEGF from FN, culture plates were coated with human plasma FN followed by addition of VEGF. VEGF was effectively bound to FN as only a small amount of VEGF spontaneously dissociated to the supernatant in the control group $(37.72 \mathrm{pg} / \mathrm{ml} \pm 23.95$; Figure 1). However, GZMB treatment elicited significant release of VEGF to the supernatant $(350 \mathrm{pg} / \mathrm{ml} \pm 61.35 ; P<0.001)$. The release of VEGF was dependent on GZMB activity as cotreatment with GZMB inhibitor (Compound 20, UBC Centre for Drug Research and Development) significantly reduced VEGF release $(147 \mathrm{pg} / \mathrm{ml} \pm 8.3 ; P<0.05)$. Thus, GZMB promotes the release of VEGF from human plasma FN.

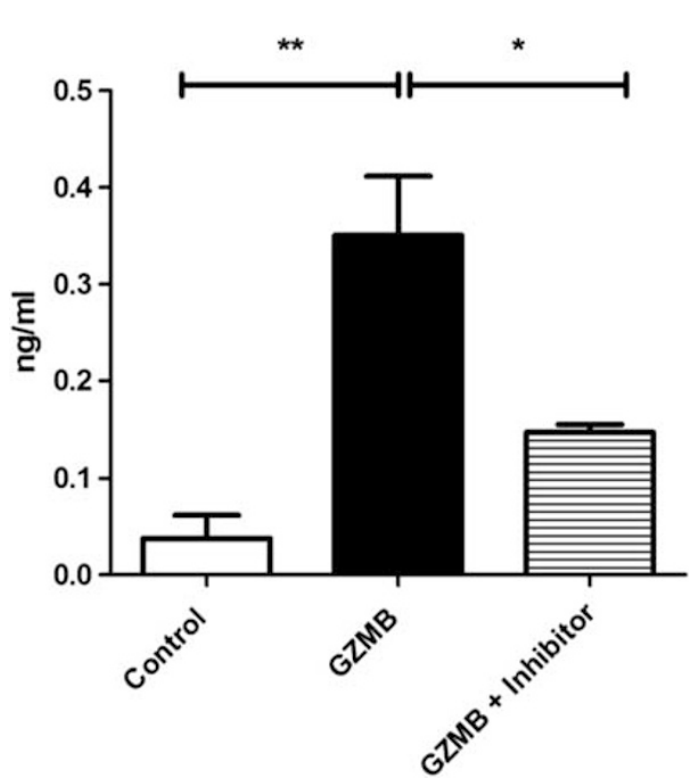

Figure 1 GZMB releases VEGF from human plasma FN. VEGF $(50 \mathrm{ng} / \mathrm{ml})$ was added to $\mathrm{FN}$-coated wells and incubated for $2 \mathrm{~h}$ at $37^{\circ} \mathrm{C}$. Unbound VEGF was removed by washing with DPBS. GZMB $(50 \mathrm{nM})$ with vehicle control (DMSO) or GZMB with inhibitor (Compound 20; $50 \mu \mathrm{M}$ ) were added to the wells and incubated for $2 \mathrm{~h}$ at $37^{\circ} \mathrm{C}$. Supernatants were removed and analyzed for VEGF by ELISA. Results are presented as mean \pm s.e.m. of $n=3$ with three triplicates per condition. ${ }^{*} P<0.05$ and ${ }^{*} P<0.01$.

\section{GZMB Releases VEGF from HUVEC Matrix}

As EC-derived ECM is more complex and may contain other pericellular matrix proteins that can bind $\mathrm{VEGF}^{28}$ it was essential to explore whether GZMB could mediate VEGF release from endogenous matrix. VEGF was added to HUVEC-derived matrix and unbound VEGF was removed by washing. Similar to experiments done on plasma FN, GZMB treatment significantly increased VEGF release from HUVEC matrix compared with control (control $135 \mathrm{pg} / \mathrm{ml} \pm 33.66$; GZMB $562 \mathrm{pg} / \mathrm{ml} \pm 39.86 ; P<0.01$; Figure 2). Inhibition of GZMB effectively reduced GZMB-mediated VEGF release $(275 \mathrm{pg} / \mathrm{ml} \pm 63.8 ; P<0.05)$. Thus, GZMB promotes VEGF release from both plasma FN and endogenously produced matrix.

\section{VEGF Is Not a Proteolytic Substrate of GZMB}

Several proteases have been demonstrated to release VEGF from the ECM by cleaving VEGF intramolecularly, generating a smaller VEGF fragment with altered biological activity. ${ }^{7,8,29,30}$ As such, the status of GZMB-released VEGF from the matrix was assessed. Plasmin was used as a positive control as it is known to cleave VEGF.7 ${ }^{7}$ To first confirm the activity and specificity of plasmin and GZMB, FN release assay was performed by adding either GZMB or plasmin to FN-coated culture plates and the supernatants were examined for the release of FN fragments by western blot. Untreated control wells gave rise to a single, faded band

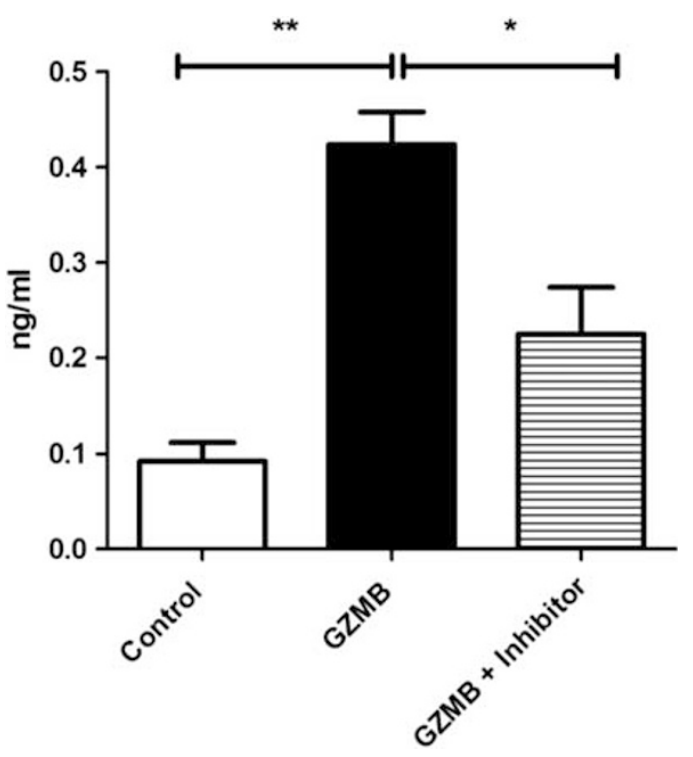

Figure 2 GZMB releases VEGF from HUVEC matrix. HUVECs were grown to confluence and maintained in serum-reduced media for 9 days. Cells were removed by adding $\mathrm{NH}_{4} \mathrm{OH}$ followed by extensive washing. Remaining ECM was incubated with VEGF $(50 \mathrm{ng} / \mathrm{ml})$ for $2 \mathrm{~h}$ at $37^{\circ} \mathrm{C}$. Unbound VEGF was removed by washing with DPBS. GZMB $(50 \mathrm{nM})$ or GZMB with inhibitor (Compound 20,50 $\mathrm{M}$ ) were added and incubated for additional $2 \mathrm{~h}$ at $37^{\circ} \mathrm{C}$. Supernatants were removed and analyzed for VEGF by ELISA. Results are presented as mean \pm s.e.m. of $n=3$ with 3 triplicates per condition. ${ }^{*} P<0.05$ and ${ }^{*} P<0.01$. 

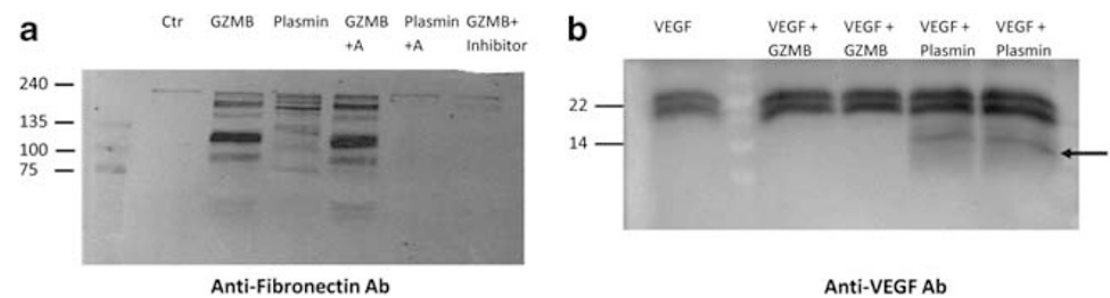

Figure 3 GZMB degrades FN but does not cleave VEGF. (a) FN-coated culture wells were treated with either GZMB or plasmin (50 nM) with or without inhibitors (aprotinin (A)) for $2 \mathrm{~h}$ at $37^{\circ} \mathrm{C}$. Supernatants were analyzed by western blotting using anti-FN Ab. (b) Same enzyme preparations used in (a) were incubated with $100 \mathrm{ng}$ VEGF in microtubes for $2 \mathrm{~h}$ at $37^{\circ} \mathrm{C}$. Samples were analyzed by western blotting using anti-VEGF Ab (arrow denotes VEGF fragment).

corresponding to the full-length FN protein that was spontaneously dissociated from the plate during the incubation period. In contrast, GZMB treatment effectively cleaved FN, yielding a number of cleavage fragments that differ in size from those generated by plasmin (Figure 3a). FN cleavage was dependent on the enzymatic activity of both proteases as addition of aprotinin and Compound 20 completely prevented FN degradation by plasmin and GZMB, respectively. Interestingly, although aprotinin is a broad-range serine protease inhibitor, it did not inhibit GZMB activity. The same enzyme preparations used for the FN release assay were used to examine VEGF proteolysis. As expected, plasmin incubation with VEGF resulted in the generation of a smaller VEGF fragment (Figure 3b, arrow). Conversely, VEGF was not cleaved by GZMB, as no VEGF fragments were observed upon GZMB treatment (Figure $3 b$ ). Thus, the release of VEGF from the ECM by GZMB is not due to an intramolecular cleavage of the VEGF molecule.

\section{GZMB-Mediated VEGF Release from FN Activates VEGFR2}

VEGFR2 dimerizes and undergoes rapid transphosphorylation in a number of tyrosine residues upon ligation with VEGF. ${ }^{5}$ We were determined to examine whether VEGF that is released from FN by GZMB retains its biological activity. HUVECs were treated with VEGF release supernatants, produced by the addition of GZMB to VEGF-bound FN as in Figure 1, and VEGFR2 activation was examined by immunoblotting for the phosphorylation of Y1214 and Y1175. Even small amounts of VEGF that spontaneously dissociate from FN activate VEGFR2 as control supernatants show distinct signal for both Y1214 and Y1175 phosphorylation, whereas HUVECs treated with media containing no VEGF ( - ve control) show no phosphorylation signal (Figure 4a). However, VEGF release supernatants that were generated because of GZMB activity caused a significant increase in VEGFR2 phosphorylation of both Y1214 and Y1175 (Figure 4a and b). VEGF released from FN treated with GZMB + inhibitor led to attenuated VEGFR2 activation. Thus, VEGF that is released from FN by GZMB is active and it can effectively lead to VEGFR2 phosphorylation in HUVECs.

\section{GZMB Mediates VEGF-Dependent Vascular Permeability In Vivo}

To test the impact of GZMB-mediated VEGF release on vascular leakage, we used the Miles assay ${ }^{31}$ by injecting mGZMB to the ear and measured Evans blue extravasation as described in the Material and Methods. To minimize the background of Evans blue dye that is present in the circulation and did not extravasate, we perfused the animal with large volume of saline $(10 \mathrm{ml})$ before analyzing the tissue. Vascular leakage at the tip of the ear was because of holding the ear with forceps to stabilize the ear during injections. Only the injection area at the base of the ear was excised with $7 \mathrm{~mm}$ punch biopsy for subsequent dye extraction. Absorbance of the extracted dye was normalized to tissue weight. The mGZMB injection resulted in increased vascular leakage compared with saline injections $(n=5)$ (Figure 5a). To further establish whether the increase in vascular leakage is dependent on VEGF activity, we coinjected mGZMB with anti-mouse neutralizing VEGF Ab. Vascular leakage was significantly reduced when mGZMB was injected with VEGF-neutralizing Ab compared with $\mathrm{mGZMB}+\operatorname{IgG}$ control injections $(n=5)$ (Figure 5b).

To confirm the source of VEGF in the in vivo experiments, untreated mouse ear sections were assessed immunohistochemically for VEGF expression. As a positive control, we used mouse kidney sections as VEGF is reported to be expressed at higher levels by podocytes within the glomerulus and to a lesser extent by the cells of the convoluted tubules; ${ }^{32}$ an observation that we confirmed in our experiments (Figure 6aiii). Within the mouse ear, VEGF is expressed in the epidermis and in the deep dermis above the cartilage tissue (Figure 6ai).This observation is in line with other studies indicating the importance of basal VEGF expression in the epidermis and dermis to maintain normal permeability barrier homeostasis ${ }^{33}$ while increased VEGF levels promote tissue edema and the development of psoriatic skin phenotype in the mouse ear. ${ }^{34}$ Immunohistochemical staining for VEGF in the ear revealed intracellular expression within the cells of the epidermis and dermis (Figure 6bi). In addition, double immunofluorescence for VEGF and FN indicates that VEGF colocalizes with FN in the deep dermis (Figure 6biii). 
a

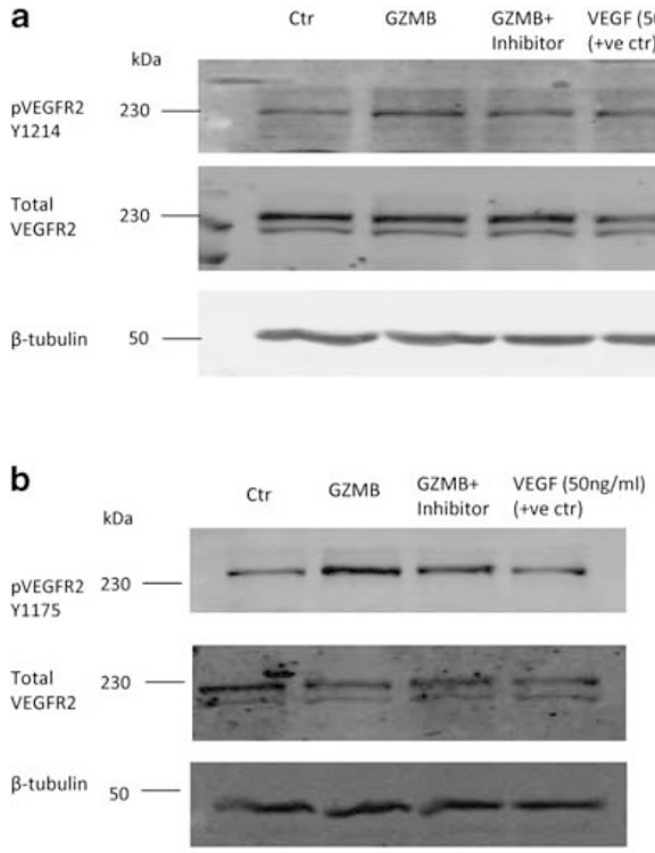

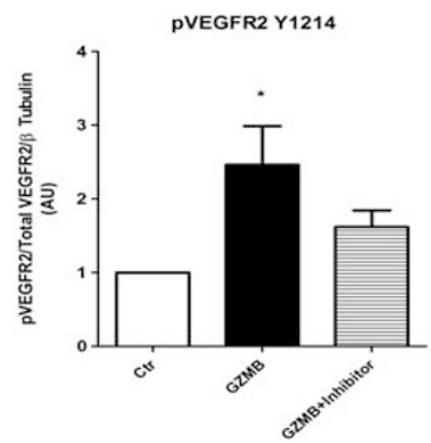

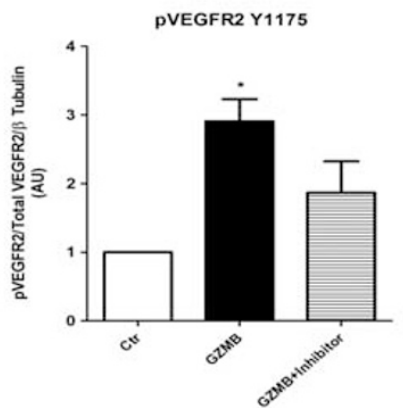

Figure 4 GZMB-mediated VEGF release from FN activates VEGFR2 in HUVECs. FN-coated wells were incubated with VEGF and treated with GZMB or GZMB with inhibitor as in Figure 1. Supernatants were removed and added to HUVEC monolayer culture for 7 min. Cell lysates were analyzed by western blotting using (a) phospho y1214 VEGFR2 Ab (pVEGFR2 y1214) and (b) phospho y1175 VEGFR2 Ab (pVEGFR2 y1175). HUVECs treated directly with VEGF $(50 \mathrm{ng} / \mathrm{ml}$ ) were used as a positive control ( + ve ctr). Total VEGFR2 and $\beta$-tubulin Abs were used as loading controls. Quantification is presented as the densitometry ratio of pVEGFR2 to total VEGFR2 normalized to $\beta$-tubulin. Results are presented as mean \pm s.e.m. of $n=3$ with three triplicates per condition. ${ }^{*} P<0.05$.
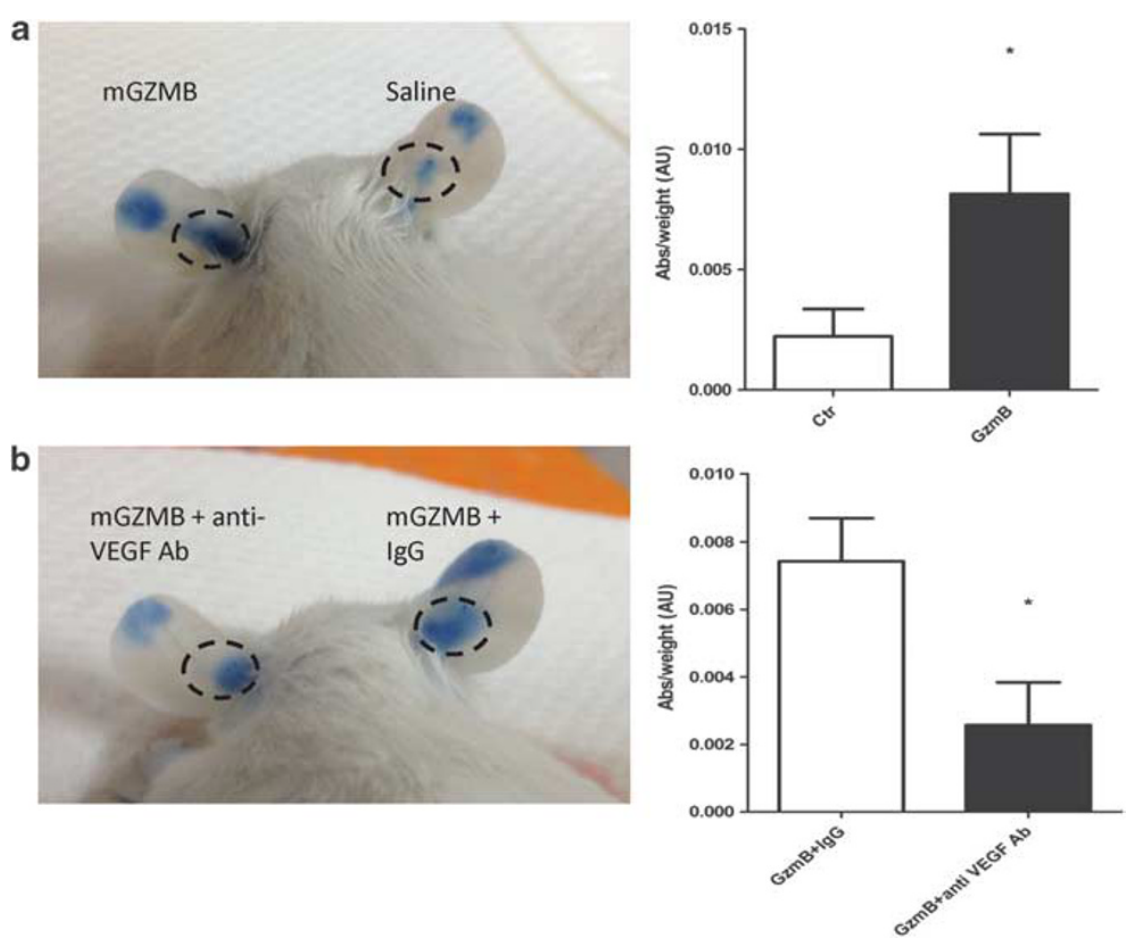

Figure 5 Mouse Gzmb induces a VEGF-dependent increase in vascular permeability in vivo. Evans blue (0.5\%) was injected via the tail vein before ear injections of (a) mGzmb (100 ng) or saline control or (b) mGzmb with anti-mouse VEGF Ab (1.5 $\mu \mathrm{g})$ or mGzmb and lgG control Ab (1.5 $\mu \mathrm{g})$. Ear tissues were excised as indicated using a $7 \mathrm{~mm}$ punch biopsy (areas in circle). Evans blue extraction was performed by drying the tissue and immersing it in formamide at $55^{\circ} \mathrm{C}$ for $24 \mathrm{~h}$. Results are presented as absorbance $(610 \mathrm{~nm})$ normalized to tissue weight $(\mathrm{mg})$. Results are presented as mean \pm s.e.m. of $n=5 .{ }^{*} P<0.05$. 
a
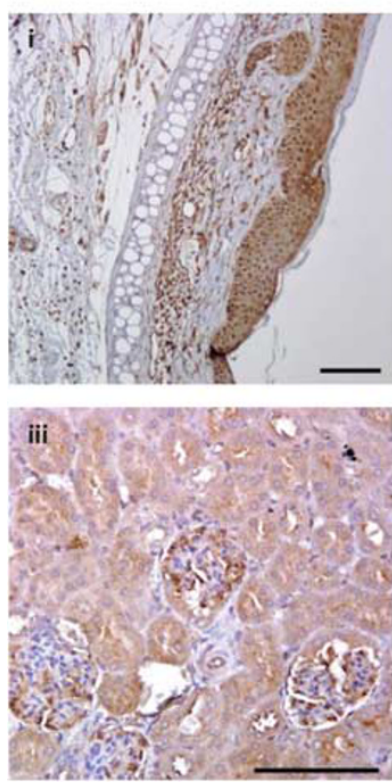
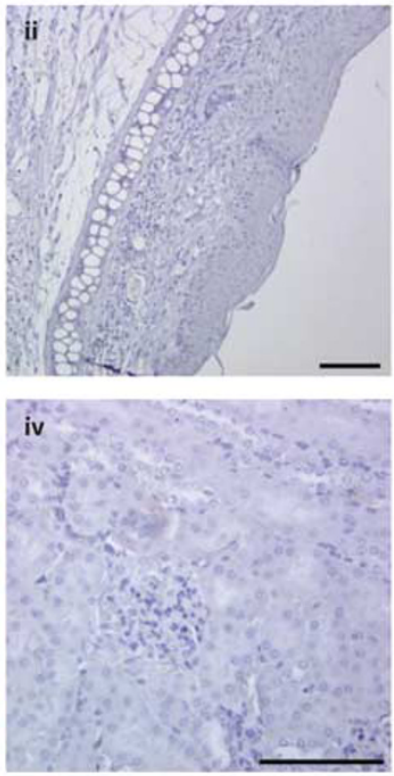

b
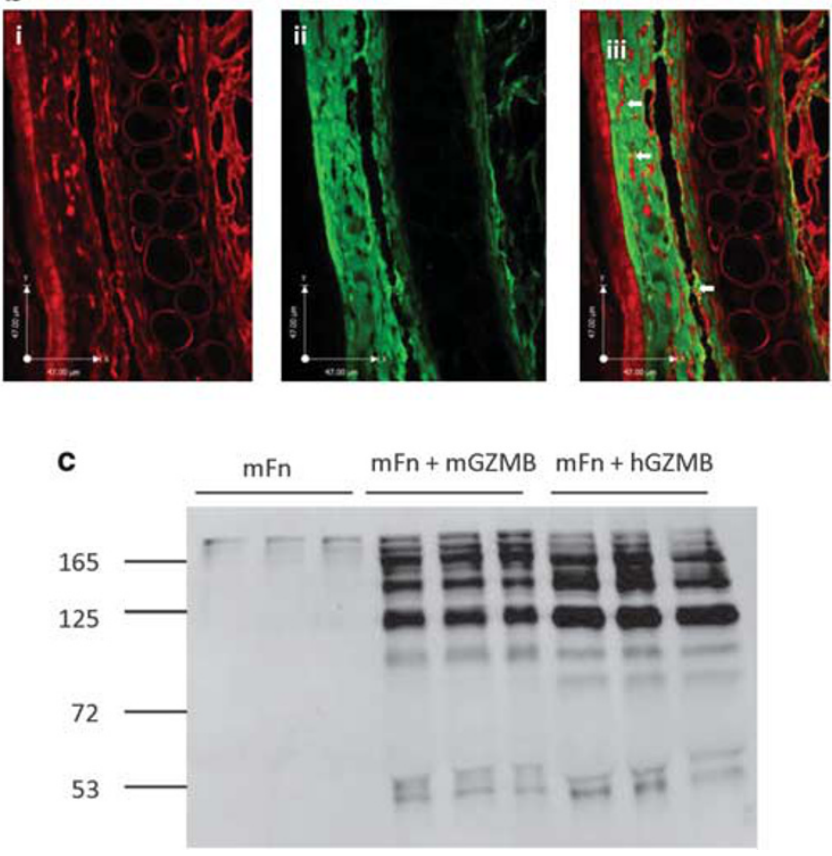

Figure 6 Endogenous VEGF expression in mouse ear and mGzmb cleavage of murine FN (mFN). (a) Untreated mouse ears were analyzed for endogenous mouse VEGF expression using immunohistochemistry with anti-mouse VEGF Ab (i). Mouse kidney sections were used as positive control for VEGF staining (iii). Ear section (ii) or kidney section (iv) stained without primary antibody were used as negative controls. Scale bar $=100 \mu$ m. (b) Double immunofluorescence using anti-mouse VEGF Ab (i, red) and anti-FN Ab (ii, green) demonstrating colocalization of VEGF and FN (iii, overlay, arrows) in the deep dermis. Scale bar $=47 \mu \mathrm{m}$. (c) Culture wells coated with $\mathrm{mFN}$ were treated with either $\mathrm{mGzmb}$ or hGZMB and incubated for $2 \mathrm{~h}$ at $37^{\circ} \mathrm{C}$. Supernatants were removed and analyzed by western blotting using anti-FN Ab.

Thus, VEGF was found to colocalize to FN in the mouse ear and serves as a source for the VEGF-dependent vascular leakage observed in the above Miles assay.

As our previous experiments included only human sources for both GZMB and FN, we wanted to confirm whether mGZMB can cleave mouse FN (mFN). FN release assay was preformed as in Figure 3 using $\mathrm{mFN}$ treated with either mGZMB or human GZMB (hGZMB). The mGZMB effectively cleaves $\mathrm{mFN}$ as a number of smaller $\mathrm{mFN}$ fragments are observed upon mGZMB treatment that are absent in the control sample (Figure 6c). The fragments that were generated by mGZMB proteolysis are similar to the fragments generated by hGZMB. However, hGZMB cleaves mFN more effectively as the resulted fragments are of higher intensity. Nevertheless, mGZMB cleaves $\mathrm{mFN}$, leading to dissociation of fragments to the supernatant.

\section{Vascular Permeability Is Reduced in GZMB-KO Mice after DTH-Induced Inflammation}

To further confirm the role of GZMB in promoting vascular permeability during inflammation, we have induced a DTH reaction on both WT and GZMB-KO mice. In this experiment, animals were first sensitized to oxazolone by topical administration on the shaved abdomen. After 7 days, ears were challenged and vascular permeability was examined after 24 and $72 \mathrm{~h}$. Vascular permeability in GZMB-KO mice

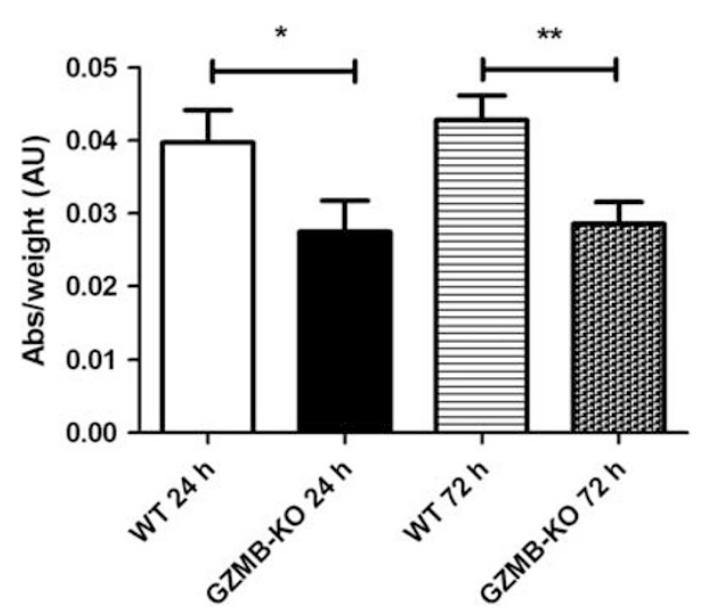

Figure 7 Vascular permeability is reduced in GZMB-KO mice after DTHinduced inflammation. WT and GZMB-KO mice were challenged with topical administration of oxazolone to both ears 7 days after initial sensitization, leading to the development of DTH inflammatory reaction in the ear. At the indicated time points, Evans blue was injected and ears were harvested followed by dye extraction to assess vascular permeability as indicated in the Materials and Methods. Results are presented as mean \pm s.e.m. $24 \mathrm{~h} \mathrm{WT}, n=8$; GZMB-KO, $n=11 ; 72 \mathrm{~h}$ WT, $n=11 ; \mathrm{GZMB}-\mathrm{KO}, n=7$. ${ }^{*} P<0.05$ and ${ }^{*} P<0.01$.

was significantly lower than in WT control at both the 24 and $72 \mathrm{~h}$ time points (Figure 7). Thus, GZMB deficiency reduced vascular permeability during DTH inflammatory reaction. 


\section{DISCUSSION}

The ECM exerts a major role in regulating growth factor bioavailability by sequestering and limiting its release. ${ }^{28}$ VEGF is a potent vascular permeability agent that is retained in the ECM by binding to FN. ${ }^{10-12}$ In the present study, GZMB increased VEGF bioavailability through FN proteolysis. GZMB-mediated VEGF release led to activation of VEGFR2 in HUVECs. GZMB-mediated vascular leakage in vivo is, in part, VEGF dependent as cotreatment of GZMB and an anti-VEGF-neutralizing $\mathrm{Ab}$ reduced vascular leakage. Importantly, we have demonstrated the role of GZMBinduced vascular permeability during the development of inflammation as GZMB deficiency reduced vascular leakage in DTH-induced inflammation in mice ears. Thus, GZMB regulates VEGF bioavailability by cleaving FN and promoting vascular leakage during inflammation in vivo.

A number of proteases were previously shown to alter VEGF-matrix interaction with significant consequences to vascular morphogenesis. Houck et $a l^{7}$ were the first to demonstrate that VEGF-expressing cells treated with plasmin gave rise to a VEGF fragment that dissociates to the supernatant and is capable of inducing vascular leakage in guinea pig skin. MMP-3 generates an unbound VEGF fragment that promotes the formation of aberrant, dilated, and leaky neovessels. ${ }^{8}$ On the other hand, MMP-resistant, matrix-bound VEGF promoted smaller vessel diameter and highly branched neovessels. ${ }^{8}$ These studies highlight the fact that VEGF activity is regulated by its interaction with the ECM, whereas proteolytic processing of VEGF and its dissociation from the matrix promotes vascular permeability and disrupts normal neovessel formation. ${ }^{35}$ As opposed to the aforementioned proteases that directly cleave VEGF, generating a smaller VEGF fragment with altered biological activity, VEGF is not a substrate of GZMB. As GZMB releases VEGF from the ECM, but does not cleave VEGF directly, we predict that GZMB alters VEGF bioavailability through the cleavage of $\mathrm{FN}$ in the ECM, leading to the release of the full-length VEGF molecule. Indeed, VEGF released from FN by GZMB retain its activity as it leads to VEGFR2 phosphorylation in HUVECs. It will be of interest to explore whether part of the FN molecule remains bound to VEGF after GZMB proteolysis or whether GZMB completely dissociates VEGF from FN.

Changes in microenvironmental levels of VEGF due to ECM processing may serve as a key mechanism for promoting vascular leakage and pathological angiogenesis in chronic inflammatory diseases. ${ }^{36}$ In an elegant study by Ozawa $e t a l,{ }^{37}$ it was demonstrated that local low levels of VEGF expression is critical for the formation of stable, nonleaky neovessels, whereas local high levels of VEGF expression resulted in enlarged, unstable, leaky capillaries that are characteristic of pathological angiogenesis. MMP-9 and MMP-2 induce tumor angiogenesis ${ }^{38}$ as well as promote inflammatory angiogenesis by releasing VEGF from the ECM. ${ }^{9}$ Moreover, these proteases were shown to promote ovarian ascites formation because of increased VEGFdependent vascular leakage. ${ }^{39}$ However, the role of MMPs in angiogenesis has been challenged by observations suggesting that MMPs are involved in neovessel regression because of the formation of antiangiogenic protein fragments via ECM proteolysis and by destabilizing EC-matrix interaction through ECM processing. ${ }^{40-43}$ Thus, it has been suggested that MMPs serve as local modulators rather than directly induce or suppress angiogenesis. ${ }^{8}$ Similarly, we have previously shown that GZMB processing of the ECM disrupts EC-matrix interaction that may pertain to its antiangiogenic role. ${ }^{26}$ Conversely, the current study may suggest that increased VEGF release by GZMB may potentially serve to induce angiogenesis. Although the direct effect of GZMBmediated VEGF release on angiogenesis has not been examined in this study, we suspect that similar to MMPs, GZMB may serve as a modulator of the angiogenic response. However, in contrast to MMPs that play a major role in normal tissue remodeling and homeostasis, elevated GZMB levels are only evident in chronic inflammatory disorders, most of which are highly associated with pathological angiogenesis, including RA, atherosclerosis, AAA, and skin pathologies. ${ }^{14,15}$ Given that GZMB increases VEGF bioavailability and induces VEGF-dependent vascular permeability, a hallmark of pathological angiogenesis, we postulate that GZMB contributes to the formation of aberrant, unstable, and leaky neovessels through ECM proteolysis and by altering microenvironmental levels of VEGF.

Previous reports indicated that hGZMB and mGZMB are different in their intracellular substrate specificity. As part of their apoptotic-inducing activities, hGZMB and mGZMB were shown to cleave procaspase- 3 more efficiently in a species-specific manner. ${ }^{44}$ Moreover, the proapoptotic protein, Bid, from both human and mouse is only cleaved by hGZMB but not by mGZMB. ${ }^{44}$ In light of these differences in substrate specificity of GZMB between species, we ensured to use mGZMB in our in vivo experiments. Importantly, we demonstrated that both hGZMB and mGZMB cleave mouse $\mathrm{FN}$, further corroborating our previous observation on the role of GZMB processing of FN in delaying wound healing in mice. ${ }^{22}$

Cumulative evidence supports the role of extracellular GZMB in altering tissue integrity and promoting inflammation through a number of mechanisms. Extracellular GZMB potentiates IL- $1 \alpha$ activity by cleaving it and generating an IL- $1 \alpha$ fragment that enhances inflammation. ${ }^{24}$ We have previously demonstrated that cleavage of fibrillin- 1 and decorin by GZMB contributes to AAA rupture in mice, ${ }^{19,20}$ whereas decorin degradation in the skin contributes to skin thinning and frailty. ${ }^{21}$ Recently, we have demonstrated that GZMB has an indirect effect on tissue remodeling as GZMB promotes the release of active TGF- $\beta$ from the ECM by cleaving a number of proteoglycans. ${ }^{25}$ The current study further corroborates the role of extracellular GZMB in altering growth factor and cytokine release and bioavailability 
during inflammation. Using the DTH mouse model, we observed a significant reduction in vascular permeability in GZMB-KO mice compared with WT control. The DTH inflammatory reaction is mediated by a number of immune cells including T cells, macrophages, and mast cells. ${ }^{45}$ Release of cytokines and proinflammatory agents promote the development of local inflammation in response to a hapten irritant that is applied 7 days after initial sensitization. Importantly, many inflammatory cells that take part in this reaction can express and release GZMB including CD8 ${ }^{+}$ $\mathrm{T}$ cells, mast cells, and macrophages. ${ }^{15,16}$ Considering the reduction in vascular permeability due to coinjection of GZMB and anti-VEGF $\mathrm{Ab}$, and the observed reduction in vascular leakage in GZMB-deficient mice during local induced inflammation, it can be postulated that extracellular release of GZMB by inflammatory cells promotes vascular permeability mediated by VEGF release from the matrix. Thus, accumulation of extracellular GZMB during chronic inflammation, as observed in a number of chronic diseases, ${ }^{14}$ may further exacerbate inflammation and delay resolution by promoting vascular permeability.

Previous studies from our laboratory and others suggest that mast cells are a major source of GZMB in the early stages of inflammation. ${ }^{21,46}$ Extracellular release of GZMB from mast cells contributes to cell detachment and disorganization of EC intercellular adherens junctions as a mechanism that may induce vascular leakage; ${ }^{46}$ however, evidence for GZMBmediated vascular leakage was not provided in the latter study. Although we cannot rule out the possibility that GZMB may contribute to vascular leakage by its direct effect on ECs in our in vivo experiments, the reduction in vascular leakage in mice that were treated with GZMB + anti-VEGFneutralizing $\mathrm{Ab}$ suggests that GZMB mediates vascular permeability predominantly through altering VEGF bioavailability.

In summary, extracellular GZMB induces VEGF release from the ECM by cleaving FN and promoting vascular permeability during inflammation in vivo. This process may serve as a novel mechanism that promotes chronic inflammation in diseases where GZMB is evident while strategies aiming to inhibit GZMB activity may attenuate vascular leakage and reduce the inflammatory response.

\section{ACKNOWLEDGMENTS}

We thank Dr Ed Pryzdial, The Centre for Blood Research, University of British Columbia, for providing plasmin for our experiments. Special thanks to Tatjana Bozin from the Genetically Engineered Models (GEM) Facility, for all your help in the in vivo experiments. AH was funded by the Vanier Canada Graduate Scholarship. $\mathrm{IH}$ is funded by the Canadian Institutes of Health Research (CIHR) Skin Research Training Scholarship. This work is funded through a grant-in-aid from CIHR.

\section{DISCLOSURE/CONFLICT OF INTEREST}

Dr David J Granville is a member of the scientific advisory board and serves as the Chief Scientific Officer to viDA Therapeutics. The remaining authors declare no conflict of interest.
1. Arroyo AG, Iruela-Arispe ML. Extracellular matrix, inflammation, and the angiogenic response. Cardiovasc Res 2010;86:226-235.

2. Nagy JA, Dvorak AM, Dvorak HF. Vascular hyperpermeability, angiogenesis, and stroma generation. Cold Spring Harb Perspect Med 2012;2:a006544.

3. Costa C, Incio J, Soares R. Angiogenesis and chronic inflammation: cause or consequence? Angiogenesis 2007;10:149-166.

4. Nagy JA, Dvorak AM, Dvorak HF. VEGF-A and the induction of pathological angiogenesis. Annu Rev Pathol 2007;2:251-275.

5. Koch S, Claesson-Welsh L. Signal transduction by vascular endothelial growth factor receptors. Cold Spring Harb Perspect Med 2012;2: a006502.

6. Lee $\mathrm{S}$, Chen $T T$, Barber $\mathrm{CL}$, et al. Autocrine VEGF signaling is required for vascular homeostasis. Cell 2007;130:691-703.

7. Houck KA, Leung DW, Rowland AM, et al. Dual regulation of vascular endothelial growth factor bioavailability by genetic and proteolytic mechanisms. J Biol Chem 1992;267:26031-26037.

8. Lee S, Jilani SM, Nikolova GV, et al. Processing of VEGF-A by matrix metalloproteinases regulates bioavailability and vascular patterning in tumors. J Cell Biol. 2005;169:681-691.

9. Ebrahem Q, Chaurasia SS, Vasanji A, et al. Cross-talk between vascular endothelial growth factor and matrix metalloproteinases in the induction of neovascularization in vivo. Am J Pathol 2010;176: 496-503.

10. Wijelath ES, Murray J, Rahman S, et al. Novel vascular endothelial growth factor binding domains of fibronectin enhance vascular endothelial growth factor biological activity. Circ Res 2002;91:25-31.

11. Wijelath ES, Rahman S, Namekata M, et al. Heparin-II domain of fibronectin is a vascular endothelial growth factor-binding domain: enhancement of VEGF biological activity by a singular growth factor/ matrix protein synergism. Circ Res 2006;99:853-860.

12. Martino MM, Hubbell JA. The 12th-14th type III repeats of fibronectin function as a highly promiscuous growth factor-binding domain. FASEB J 2010;24:4711-4721.

13. Martino MM, Tortelli F, Mochizuki $M$, et al. Engineering the growth factor microenvironment with fibronectin domains to promote wound and bone tissue healing. Sci Transl Med 2011;3:100ra89.

14. Hendel A, Hiebert PR, Boivin WA, et al. Granzymes in age-related cardiovascular and pulmonary diseases. Cell Death Differ 2010;17: 596-606.

15. Boivin WA, Cooper DM, Hiebert PR, et al. Intracellular versus extracellular granzyme $B$ in immunity and disease: challenging the dogma. Lab Invest 2009;89:1195-1220.

16. Hiebert PR, Granville DJ. Granzyme B in injury, inflammation, and repair. Trends Mol Med 2012;18:732-741.

17. Kondo $H$, Hojo $Y$, Tsuru $R$, et al. Elevation of plasma granzyme $B$ levels after acute myocardial infarction. Circ J 2009;73:503-507.

18. Kummer JA, Tak PP, Brinkman BM, et al. Expression of granzymes $A$ and $B$ in synovial tissue from patients with rheumatoid arthritis and osteoarthritis. Clin Immunol Immunopathol 1994;73:88-95.

19. Ang LS, Boivin WA, Williams SJ, et al. Serpina3n attenuates granzyme B-mediated decorin cleavage and rupture in a murine model of aortic aneurysm. Cell Death Dis 2011;2:e209.

20. Chamberlain CM, Ang LS, Boivin WA, et al. Perforin-independent extracellular granzyme B activity contributes to abdominal aortic aneurysm. Am J Pathol 2010;176:1038-1049.

21. Hiebert PR, Boivin WA, Abraham T, et al. Granzyme B contributes to extracellular matrix remodeling and skin aging in apolipoprotein $E$ knockout mice. Exp Gerontol 2011;46:489-499.

22. Hiebert PR, Wu D, Granville DJ. Granzyme B degrades extracellular matrix and contributes to delayed wound closure in apolipoprotein $\mathrm{E}$ knockout mice. Cell Death Differ 2013;20:1404-1414.

23. Omoto Y, Yamanaka K, Tokime K, et al. Granzyme B is a novel interleukin-18 converting enzyme. J Dermatol Sci 2010;59:129-135.

24. Afonina IS, Tynan GA, Logue SE, et al. Granzyme B-dependent proteolysis acts as a switch to enhance the proinflammatory activity of IL-1alpha. Mol Cell 2011;44:265-278.

25. Boivin WA, Shackleford M, Vanden Hoek A, et al. Granzyme B cleaves decorin, biglycan and soluble betaglycan, releasing active transforming growth factor-beta1. PLoS One 2012;7:e33163.

26. Hendel A, Granville DJ. Granzyme B cleavage of fibronectin disrupts endothelial cell adhesion, migration and capillary tube formation. Matrix Biol 2013;32:14-22. 
27. Willoughby CA, Bull HG, Garcia-Calvo M, et al. Discovery of potent, selective human granzyme B inhibitors that inhibit CTL mediated apoptosis. Bioorg Med Chem Lett 2002;12:2197-2200.

28. Macri L, Silverstein D, Clark RA. Growth factor binding to the pericellular matrix and its importance in tissue engineering. Adv Drug Deliv Rev 2007;59:1366-1381.

29. Keyt BA, Berleau LT, Nguyen HV, et al. The carboxyl-terminal domain (111-165) of vascular endothelial growth factor is critical for its mitogenic potency. J Biol Chem 1996;271:7788-7795.

30. Kurtagic E, Jedrychowski MP, Nugent MA. Neutrophil elastase cleaves VEGF to generate a VEGF fragment with altered activity. Am J Physiol Lung Cell Mol Physiol 2009;296:L534-L546.

31. Miles AA, Miles EM. Vascular reactions to histamine, histamineliberator and leukotaxine in the skin of guinea-pigs. J Physiol 1952;118:228-257.

32. Maharaj AS, Saint-Geniez M, Maldonado AE, et al. Vascular endothelial growth factor localization in the adult. Am J Pathol 2006;168:639-648.

33. Elias PM, Arbiser J, Brown BE, et al. Epidermal vascular endothelia growth factor production is required for permeability barrier homeostasis, dermal angiogenesis, and the development of epidermal hyperplasia: implications for the pathogenesis of psoriasis. Am J Pathol 2008;173:689-699.

34. Xia YP, Li B, Hylton D, et al. Transgenic delivery of VEGF to mouse skin leads to an inflammatory condition resembling human psoriasis. Blood 2003;102:161-168.

35. Ferrara N. Binding to the extracellular matrix and proteolytic processing: two key mechanisms regulating vascular endothelial growth factor action. Mol Biol Cell 2010;21:687-690.

36. Weis SM, Cheresh DA. Pathophysiological consequences of VEGFinduced vascular permeability. Nature 2005;437:497-504.

37. Ozawa CR, Banfi A, Glazer NL, et al. Microenvironmental VEGF concentration, not total dose, determines a threshold between normal and aberrant angiogenesis. J Clin Invest 2004;113:516-527.
38. Bergers G, Brekken R, McMahon G, et al. Matrix metalloproteinase-9 triggers the angiogenic switch during carcinogenesis. Nat Cell Biol 2000;2:737-744.

39. Belotti $\mathrm{D}$, Paganoni $\mathrm{P}$, Manenti $\mathrm{L}$, et al. Matrix metalloproteinases (MMP9 and MMP2) induce the release of vascular endothelial growth factor (VEGF) by ovarian carcinoma cells: implications for ascites formation. Cancer Res 2003;63:5224-5229.

40. Aplin AC, Zhu WH, Fogel E, et al. Vascular regression and survival are differentially regulated by MT1-MMP and TIMPs in the aortic ring model of angiogenesis. Am J Physiol Cell Physiol 2009;297: C471-C480.

41. O'Reilly MS, Wiederschain D, Stetler-Stevenson WG, et al. Regulation of angiostatin production by matrix metalloproteinase-2 in a model of concomitant resistance. J Biol Chem 1999;274: 29568-29571.

42. Pozzi $\mathrm{A}$, Moberg PE, Miles LA, et al. Elevated matrix metalloprotease and angiostatin levels in integrin alpha 1 knockout mice cause reduced tumor vascularization. Proc Natl Acad Sci USA 2000;97:22022207.

43. Hamano $Y$, Zeisberg $M$, Sugimoto $H$, et al. Physiological levels of tumstatin, a fragment of collagen IV alpha3 chain, are generated by MMP-9 proteolysis and suppress angiogenesis via alphaV beta3 integrin. Cancer Cell 2003;3:589-601.

44. Casciola-Rosen L, Garcia-Calvo M, Bull HG, et al. Mouse and human granzyme $\mathrm{B}$ have distinct tetrapeptide specificities and abilities to recruit the bid pathway. J Biol Chem 2007;282:4545-4552.

45. Honda T, Egawa G, Grabbe $\mathrm{S}$, et al. Update of immune events in the murine contact hypersensitivity model: toward the understanding of allergic contact dermatitis. J Invest Dermatol 2013;133: 303-315.

46. Pardo J, Wallich R, Ebnet $\mathrm{K}$, et al. Granzyme B is expressed in mouse mast cells in vivo and in vitro and causes delayed cell death independent of perforin. Cell Death Differ 2007;14:1768-1779. 